\title{
Self-Seeded RSOAs WDM PON field trial for business and mobile fronthaul applications
}

\author{
F. Saliou ${ }^{(1)}$, G. Simon $^{(1)}$, P. Chanclou ${ }^{(1)}$, M. Brunero ${ }^{(2)}$, L. Marazzi $^{(2)}$, P. Parolari $i^{(2)}$, M. Martinelli $^{(2)}$, R.

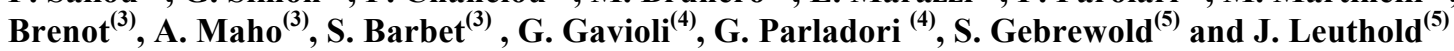 \\ (1) Orange Labs, 2 avenue Pierre Marzin, 22307 Lannion,France, fabienne.saliou@orange.com \\ (2) Politecnico di Milano, Dip. Elettronica Informazione e Bioingegneria, Via Ponzio 34/5,20133 Milano, Italy \\ (3) III-V Labs-Route de Nozay F-91461 Marcoussis Cedex, France \\ (4) Optics Division, Alcatel-Lucent Italia, Vimercate MB, 20871, Italy \\ (5) Institute of Electromagnetic Fields (IFH), ETH Zurich, Gloriastrasse 35, Zürich, Switzerland
}

\begin{abstract}
GEth, CPRI and $10 \mathrm{Gbit} / \mathrm{s}$ transmissions are experimented using amplified and standard self-seeded RSOA WDM PON systems. A field trial setup was exploited to test the system performance in terms of reach and optical budget.

OCIS codes: (060.4510) Optical communications; (060.4264) Networks, wavelength assignment
\end{abstract}

\section{Introduction}

Wavelength division multiplexing passive optical networks (WDM PON) has been extensively studied with several technological options [1]. The FP7 ERMES project (Embedded Resonant and ModulabIE Self-Tuning Laser Cavity for Next Generation Access Network Transmitter) focused on the realization of a colorless WDM PON transmitter based on self-seeded reflective semiconductor optical amplifiers (RSOAs). ERMES approach has the advantage to provide an automatic and passive mechanism for the wavelength assignment, as the transmitter self-tunes on the channel of the WDM filter which is connected to the RSOA via the drop fiber. So far, results proved the capabilities of this system with $10 \mathrm{Gbit} / \mathrm{s}$ transmission over tens of kilometers of standard single mode fiber (SSMF) and very long self-seeded laser cavities [2]. In this paper we demonstrate, within a field trial setup, the successful exploitation of the proposed WDM PON system based on amplified self-seeded and regular selfseeded with Gigabit-Ethernet and common public radio interface (CPRI) traffics, respectively for business users and mobile fronthaul [3], as well as the possibility to obtain error free transmission at $10 \mathrm{Gbit} / \mathrm{s}$.

\section{Field trial setup}

The field trial consists in the experimentation of the proposed WDM PON system over the city of Lannion (France) fiber rings. Each loop of the ring has a length of $18600 \mathrm{~m}$ and insertion losses around $12 \mathrm{~dB}$, mainly due to the high number of connectors in the ring.

The experimental setup is depicted in Figure 1 for an amplified self-seeded architecture [4]. The WDM PON optical distribution network (ODN) has a classical configuration with simply one multiplexer (MUX) at the remote node (RN) and one demultiplexer (DMUX) at the central office (CO) respectively placed at the terminations of the field trial fiber ring. The two multiplexers are flat-top with $200 \mathrm{GHz}$ spacing and 4 channels. At the user premises (RRH or Business access point), the transmitter consists of a packaged high polarization dependant gain (PDG) RSOA (more than $25 \mathrm{~dB}$ small signal gain) with a $45^{\circ}$ Faraday Rotator (FR45) at its output. The RSOA is directly modulated with a bias current optimized between $125 \mathrm{~mA}$ and $145 \mathrm{~mA}$ according to the desired cavity length to achieve. At the CO, which can be either the base band unit (BBU) hotel or an Edge Node Router (respectively for mobile fronthaul or business networks), the receiver is based on an avalanche photodiode (APD)

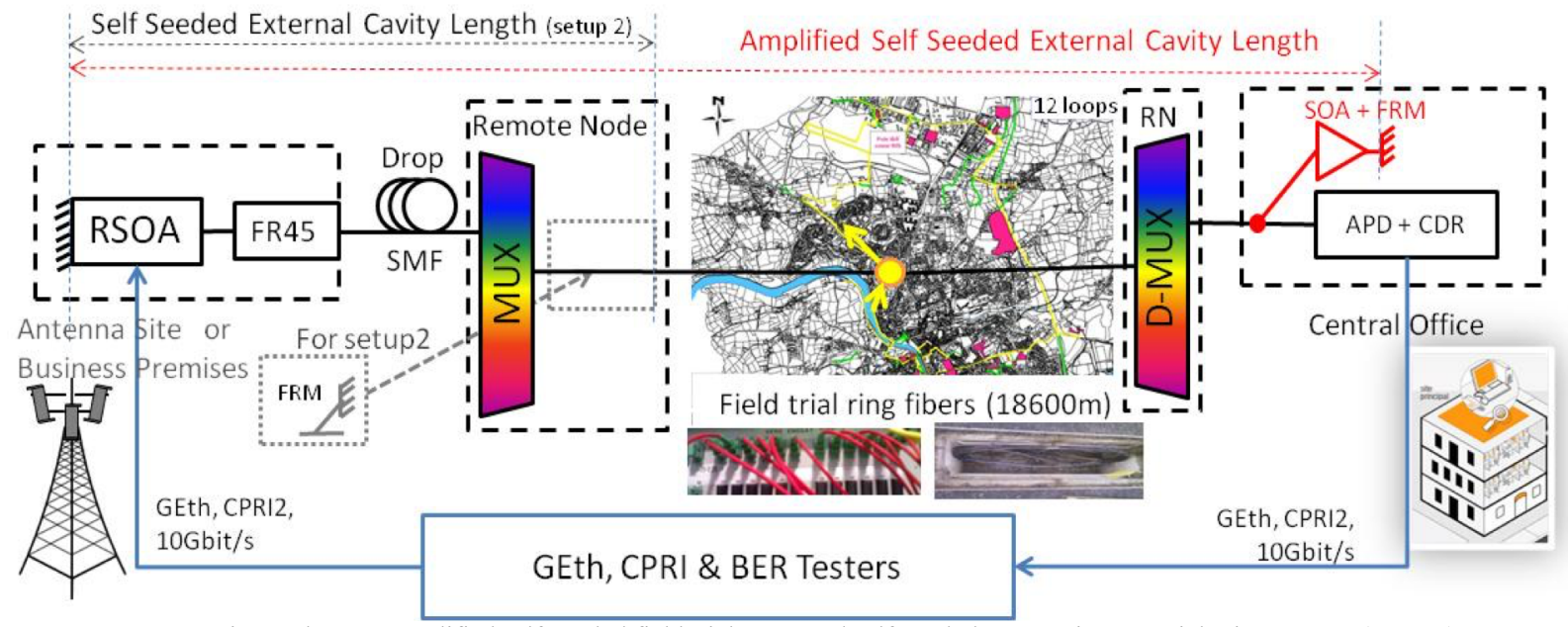

Fig. 1: Experimental setup: amplified self-seeded field trial setup and self-seeded setup using a partial mirror at RN (setup 2) 
(C) 2015 Optical Society of America. One print or electronic copy may be made for personal use only. Systematic reproduction and distribution, duplication of any material in this paper for a fee or for commercial purposes, or modifications of the content of this paper are prohibited. https://doi.org/10.1364/OFC.2015.M2A.2

followed by a commercially available clock and data recovery (CDR), providing also electronic dispersion compensation (EDC) for the $10 \mathrm{Gbit} / \mathrm{s}$ transmissions. To realize an amplified self-seeded architecture, a low-PDG SOA is associated to a Faraday rotator mirror (FRM) and inserted close to the receiver with a 80/20 coupler. Thus, an external cavity laser is created along the entire network: from the RSOA to the FRM. The RSOA outputs a wide band O-Band amplified spontaneous emission which is then spectrally sliced by the MUX, according to the connected channel (here $1325.8 \mathrm{~nm}$ ). This signal propagates through the network fiber and is amplified and back reflected by the SOA and the FRM. After several round trips in the cavity, an amplified self-seeded laser reaches its steady state condition [4].

Tab. 1: GEth and CPRI2 test results vs fiber reach

\begin{tabular}{|c|c|c|c|c|}
\hline $\begin{array}{c}\text { Cavity } \\
\text { Length }\end{array}$ & $\begin{array}{c}\text { 1 Field } \\
\text { Trial Loop }\end{array}$ & $\mathbf{2 5} \mathbf{~ k m}$ & $\mathbf{3 5} \mathbf{~ k m}$ & $\mathbf{5 0 ~} \mathbf{~ m}$ \\
\hline & & Error & Error & \\
GEth & Error Free & Free & Free & $5.10^{-9}$ \\
\hline & & Error & Error & \\
CPRI2 & Error Free & Free & Free & $1.10^{-4}$ \\
\hline
\end{tabular}

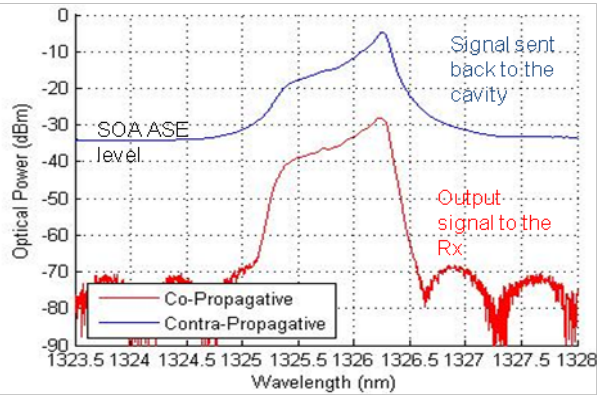

Fig. 2: Amplified Self-seeded optical spectra copropagating (red) and counterpropagating (blue), meas. at the DMUX output.

During the field trial, Giga Ethernet (GEth) (1518 bytes frame including pseudo random bit sequence (PRBS) $2^{7}$ 1 test sequence) and CPRI2 (1.228 Gbit/s, equivalent to PRBS $\left.2^{31}-1\right)$ traffics was generated by GEth and CPRI testers. Results of the transmission performance are summarized in Table 1, showing that error free transmissions were realized for up to $35 \mathrm{~km}$ of SSMF. The maximum reached transmission length was $50 \mathrm{~km}$, due to optical budget (OB) limitations. Moreover only 1 field trial loop of 18,6 $\mathrm{km}$ was achievable because of its high insertion losses.

Figure 3 and Figure 4 present $2.5 \mathrm{Gbit} / \mathrm{s}$ results from a BER test bed respectively obtained with $2^{7}-1$ and $2^{31}-1$ PRBS sequences. With $25 \mathrm{~km}$ and 1 field trial loop of fiber, error free $\left(\mathrm{BER}<10^{-9}\right)$ transmissions were realized for a short PRBS sequence $\left(2^{7}-1\right)$ at $2.5 \mathrm{Gbit} / \mathrm{s}$. However to achieve higher reach or longer pattern sequences $\left(2^{31}-1\right)$ as recommended for CPRI3, the BER was limited to $4.10^{-9}$ at best ( $25 \mathrm{~km}$ curve, gray squares). Thus, the use of a proper forward error correction (FEC) code is recommended. Furthermore, for a $10 \mathrm{Gbit} / \mathrm{s}$ transmission with a $2^{7}$ 1 PRBS, the BER was limited to $4.10^{-4}$ with $10 \mathrm{~km}$ of fiber cavity. Thanks to the presence of a second gain element, amplified self-seeded allows for very long cavity lengths, which obviously present a very high number of modes and consequently a high relative intensity noise (RIN) with respect to shorter cavities. Comparing $2^{7}-1$ PRBS and $2^{31}-1$ PRBS results, a patterning effect has also to be noticed and can be related to the combined effect of RSOA bandwidth limitations and the cancellation-remodulation condition at the RSOA.

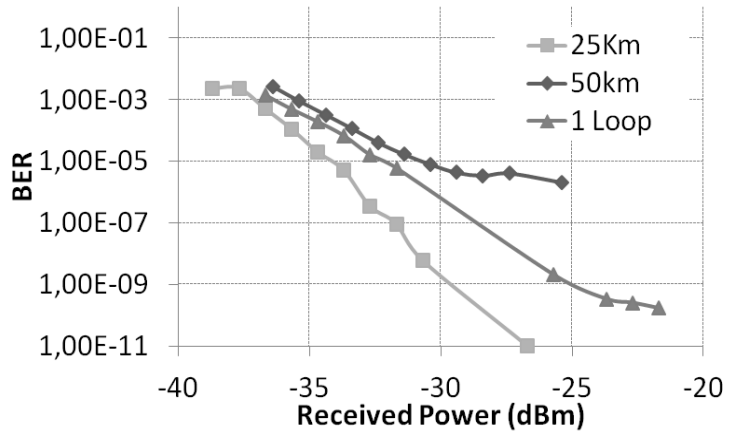

Fig. 3: $2.5 \mathrm{~Gb} / \mathrm{s}$ BER results vs. received power with $25 \mathrm{~km}$ (gray squares), $50 \mathrm{~km}$ (black diamonds) SSMF and 1 field trial loop (18600 m, gray triangles) for a 27-1 PRBS

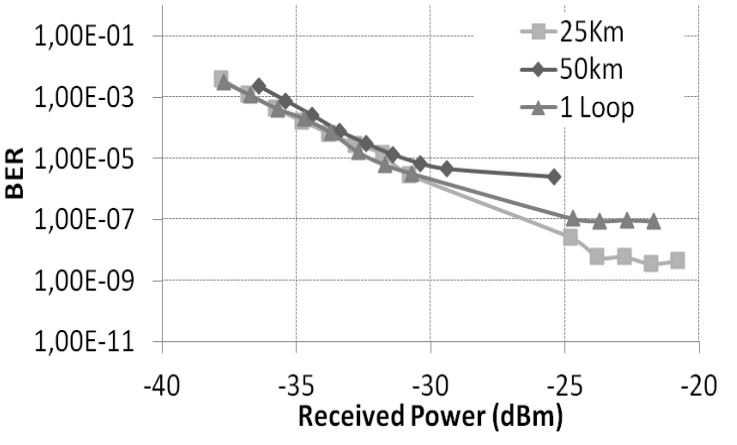

Fig. 4: $2.5 \mathrm{~Gb} / \mathrm{s}$ BER results vs received power with $25 \mathrm{~km}$ (gray squares), $50 \mathrm{~km}$ (black diamonds) SSMF and 1 field trial loop (18600m, gray triangles) for a $2^{31}-1$ PRBS

In order to avoid the ASE noise of the SOA in amplified self-seeded configuration and bridging long fiber distance while relying on shorter fiber cavities, a regular self-seeded architecture was also experimented, the cavity length in fact is defined by the drop fiber only. Here, the SOA is removed and the partial mirror (a 90/10 splitter with a FRM) is placed at the RN, on the common port of the Multiplexer (setup 2 of Figure 1). For this architecture, a 32 channels, $100 \mathrm{GHz}$ spacing Gaussian shaped AWG has been exploited, which previously allowed $10 \mathrm{~Gb} / \mathrm{s}$ transmission [2]. No DMUX was implemented in this experimental setup.

Table 2 summarizes the results obtained for the experimentation of GEth and CPRI 2 traffics over the field trial fiber rings and for several cavity lengths $(10 \mathrm{~m}, 300 \mathrm{~m}$ and $1 \mathrm{~km})$. 
Tab. 2: Self-seeded error free achievements with GEth or CPRI2 for various cavity and feeder fiber lengths

\begin{tabular}{|c|c|c|c|}
\hline $\begin{array}{c}(1 \text { loop }= \\
18600 \mathrm{~m})\end{array}$ & $\mathbf{1 0 m}$ cavity & $\begin{array}{c}\mathbf{3 0 0 m} \\
\text { cavity }\end{array}$ & $\begin{array}{c}\mathbf{1 k m} \\
\text { cavity }\end{array}$ \\
\hline B2B & $\begin{array}{c}\text { GEth } \\
\text { CPRI2 }\end{array}$ & $\begin{array}{c}\text { GEth } \\
\text { CPRI2 }\end{array}$ & GEth \\
\hline 1 loop & GEth & GEth & GEth \\
\hline 2 loops & GEth & GEth & OB limit \\
\hline
\end{tabular}

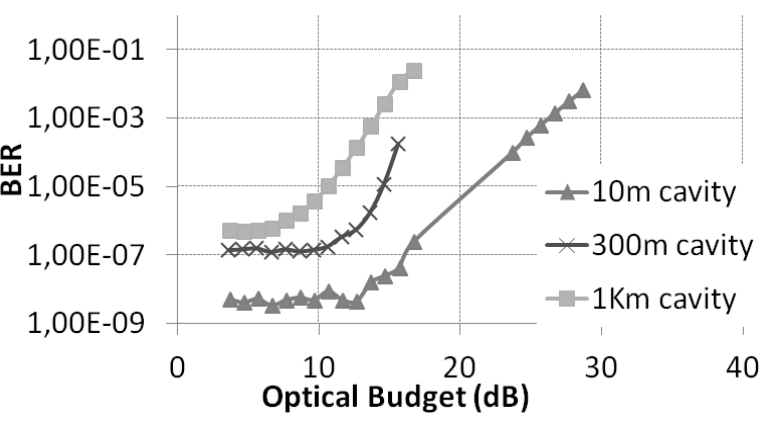

Fig. 5: BER results according to cavity length at $2.5 \mathrm{Gbit} / \mathrm{s}\left(2^{31}-1\right.$ PRBS) for back-to-back transmission.

With the self-seeded architecture, GEth was transmitted successfully for each cavity length and over at least one field trial fiber ring. Transmission errors occurred for 2 loops and $1 \mathrm{~km}$ cavity length because of the limitation of the optical budget, due to field trial fiber ring very high losses. CPRI2 transmission reach (i.e. optical budget) was reduced since error free with a $2^{31}-1$ PRBS is required and, as alredy stated, patterning effect was observed on measured BER curves.

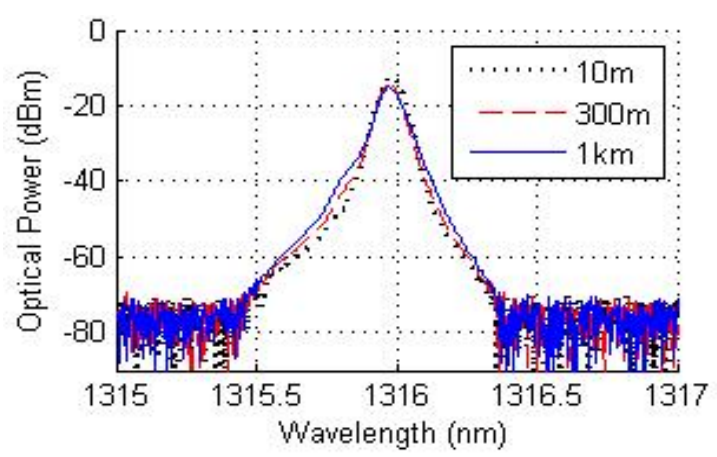

Fig. 6: Self-seeded optical spectra with the Gaussian AWG and several cavity lengths.

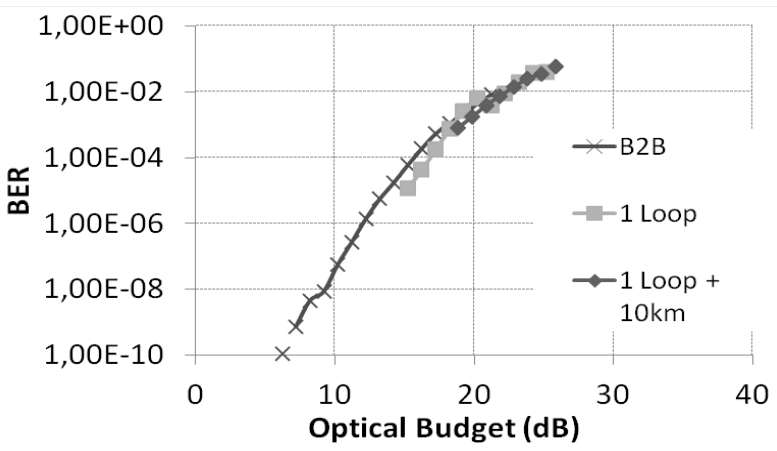

Fig.7: 10-Gbit/s BER results according to the transmission fiber length for a 10 -m cavity length $\left(2^{7}-1\right.$ PRBS $)$ vs OB.

Figure 5 shows back-to-back BER results and Figure 6 presents the corresponding optical spectra, at $2.5 \mathrm{Gbit} / \mathrm{s}$ with $2^{31}-1$ PRBS when varying the cavity length. Penalties due to longer cavity lengths are observed due to the higher RIN associated with the increasingly higher number of cavity modes. Nevertheless the exploitation of a FEC with $10^{-4}$ BER limit, would allow error free tranmission for $\mathrm{OB}$ of $25.7 \mathrm{~dB}, 15.6 \mathrm{~dB}$ and $13.7 \mathrm{~dB}$ respectively for $10 \mathrm{~m}, 300 \mathrm{~m}$ and $1 \mathrm{~km}$ cavity lengths.

Figure 7 presents the BER results at $10 \mathrm{Gbit} / \mathrm{s}$ for a $10 \mathrm{~m}$ cavity in back to back, after 1 loop of the field trial fiber ring and 1 loop and $10 \mathrm{~km}$ of SSMF. Error free transmission was realized for back to back. Moreover, as expected, with O-band operation, no penalties due to chromatic dispersion were observed. An OB of $18 \mathrm{~dB}$ can be achieved using a proper FEC $\left(10^{-4} \mathrm{BER}\right.$ limit).

\section{Conclusions}

A WDM PON system based on amplified self- seeded RSOAs was experimented in a field trial configuration. Very long external cavity lasers were realized and GEth and CPRI transmissions were demonstrated as well as 2.5 $\mathrm{Gbit} / \mathrm{s}$ BER error free results. $10 \mathrm{Gbit} / \mathrm{s}$ transmissions were realized with a standard self-seeded architecture with $28.6 \mathrm{~km}$ reach.

Optical budget, reach, and bit rate achievements showed the great capabilities of the proposed self-tuning DWDM technology for business and mobile fronthaul networks with typical reach of $20 \mathrm{~km}$ and a few hundred meters between the network termination and the remote node.

\section{Acknowledgements}

This work has been supported by the FP7 EU Project ERMES, under grant agreement $\mathrm{n}^{\circ} 288542$.

\section{References}

[1] P. Vetter, "Next Generation Optical Access Technologies," Proc. ECOC2012, tutorial session, Amsterdam (2012), p1-42

[2] P. Parolari et al, "10-Gb/s Operation of a Colorless Self-Seeded Transmitter Over More Than 70 km of SSMF", IEEE Photon.

Technol. Lett, Vol. 26, no. 6, p. 599, 2014

[3] P. Chanclou, et al., "Optical fiber solution for mobile fronthaul to achieve cloud radio access network," in Proc. Future Network Mobile Summit 2013, Lisbone, Portugal, pp. 1-11F.

[4] G. Simon et al, "70km external cavity DWDM sources based on O-band self seeded RSOAs for transmissions at 2.5Gbit/s", Proc OFC2014, San Francisco, CA, USA, pW.3.G.5 